\title{
The effects of sex and exposure to inescapable shock on shock-elicited fighting in albino rats
}

\author{
D. CHRIS ANDERSON, CHARLES R. CROWELL, \\ J. VICTOR LUPO, and THERESA SARMINA \\ University of Notre Dame, Notre Dame, Indiana 46556
}

\begin{abstract}
A 2 by 2 factorial design was employed to investigate the effects of exposure to inescapable shock vs. no shock on the subsequent shock-elicited fighting behavior of male and female rats. Results indicated that males fought reliably more than females in response to brief test shocks. Also, prior exposure to inescapable shock was observed to reduce markedly the degree of shockelicited fighting for both male and female rats during initial test sessions. In addition, measurements of responsivity to shock were observed to decrease for shock-treated subjects as a function of continued exposure to inescapable shock during the shock-treatment phase. Taken together, these findings indicate that although sex can be a powerful determinant of degree of shock-elicited fighting in albino rats, the debilitating effect of exposures to inescapable shock does not depend upon this factor. Furthermore, the observations of behavior during shock treatment itself tentatively suggest that the marked reduction in fighting produced by this variable may be due, in part, to a behavioral change accompanying exposure to inescapable shock.
\end{abstract}

Exposure to inescapable shock has been shown to suppress markedly the subsequent shock-elicited fighting behavior of male albino rats (Anderson, Murcurio, \& Mahoney, 1970; Maier, Anderson, \& Lieberman, 1972; Payne, Anderson, \& Murcurio, 1970; Powell \& Creer, 1969; Powell, Francis, Francis, \& Schneiderman, 1972; Tondat, 1974). For the most part, investigators have ignored questions regarding the extent to which this finding generalizes to female rat subjects. One notable exception is the study by Powell et al. (1972) in which a fightinginterference effect following exposure to inescapable shock was reported for a combined population of male and female rats. Unfortunately, however, this study was not designed to permit separate comparisons of the effect of shock treatments on each sex involved. This oversight followed from the authors' assumption that gender was not an important variable in determining degree of shock-elicited fighting in albino rats, a contention that was based upon the results of several prior studies showing little, if any, sex effect on degree of shock-elicited fighting in this strain (Milligan, Powell, \& Borasio, 1973; Powell, Silverman, Francis, \& Schneiderman, 1970; Ulrich \& Azrin, 1962).

The possibility of discounting sex differences in the fighting behavior of albino rats should be regarded

Some of this research was supported by the University of Notre Dame O'Brien Fund. Parts of this paper were presented at the Midwestern Psychological Association, 1975, Chicago. Requests for reprints should be sent to D. Chris Anderson or Charles R. Crowell, Department of Psychology, University of Notre Dame, Notre Dame, Indiana 46556. with skepticism for two reasons. First, there is considerable evidence that gender-related fighting differences do occur in strains other than the albino rat. In studies with the hooded, Long-Evans strain, males have been found to fight more in response to shock than females (Connor \& Levine, 1969; Hutchinson, Ulrich, \& Azrin, 1965; Milligan et al., 1973). And secondly, careful comparison of the procedures employed in the studies that do and do not demonstrate sex-related differences in fighting behavior reveals disparities in potentially important variables such as parameters of the test shock. The possibility exists, therefore, that the failures to find sex differences in the fighting behavior of albino rats may be due as much to peculiar methodological details as to subject strain. Accordingly, the present study was designed to reevaluate the degree of shock-elicited fighting obtained in male and female albino rats. A second purpose of this study was to assess separately the extent to which exposure to inescapable shock subsequently affects degree of shock-elicited fighting in each sex. Thus, the design of the study involved a completely crossed combination of subject gender and the presence or absence of shock treatment.

\section{METHOD}

\section{Subjects}

The subjects were 40 naive, Sprague-Dawley rats obtained from Harlan Industries, Cumberland, Indiana. Half of the subjects were male and the other half female, and all were approximately 110 days of age at the beginning of the experiment. From the outset, each subject was weighed daily and given free access to food and water throughout the study. All rats were individually housed under conditions of a 12-h light-dark cycle. The treat- 
ment and testing occurred at approximately the same time in the cycle each day, beginning $10 \mathrm{~h}$ following light onset and approximately $8 \mathrm{~h}$ after feeding.

\begin{abstract}
Apparatus
Treatment. Shock treatment was administered in a $17.7 \times$ $25.3 \times 20.1 \mathrm{~cm}$ (inside dimensions) chamber composed of stainless steel walls and a grid floor consisting of $0.8-\mathrm{cm}$ brass rods spaced $1.7 \mathrm{~cm}$ apart, center to center. The source of the shock was a tube-regulated, dc apparatus (Campbell \& Teghtsoonian, 1958) that maintained a constant current over a load range from 0 to $3 \mathrm{~m} \Omega$. The output of this device was adjusted to deliver $1.25 \mathrm{~mA}$ to the grid through a Lehigh Valley shock scrambler. In an effort to obtain a gross indication of subject reactivity to treatment shock, the current through each subject was monitored continuously by a multimeter (Triplett Model 630-A, 20,000 $\Omega / \mathrm{dc}$ ) connected in series with the grid floor. In general, movements in the presence of shock were reflected in fluctuations in the current reading on the meter. The treatment chamber was housed in a sound-deadened, darkened, ventilated refrigerator shell. All treatment contingencies were controlled by supporting equipment located elsewhere.

Testing. Shock-elicited fighting tests were conducted in an acrylic chamber with inside dimensions of $15.2 \times 15.2 \times$ $30.4 \mathrm{~cm}$. The floor and front inside wall of this chamber were composed of $0.4-\mathrm{cm}$ brass rods, spaced $1.25 \mathrm{~cm}$ apart, center to center. The wall rods were arranged vertically along the inside surface of a Plexiglas sheet. The remaining three walls were lined with isolated sheets of stainless steel arranged in such a way that all grid and metal surfaces could be electrified independently. This chamber was housed in a sound-deadened, ventilated refrigerator shell with a clear plastic window that provided for observation of fighting. During testing, a 7.5-W bulb illuminated the interior of the refrigerator shell that was located in a darkened room. The brief shocks used to elicit fighting in this phase were generated by the same source and scrambler arrangement described above, but were measured at $3 \mathrm{~mA}$ with a dummy load of $29 \mathrm{k} \Omega$ substituted in place of the rat. This intensity was selected on the basis of preliminary research indicating that optimum levels of fighting occurred with this shock.
\end{abstract}

\section{Procedure}

Following their arrival from the supplier, the rats were weighed and handled for 4 consecutive days prior to the beginning of treatment. Within each sex group, the subjects were assigned to shock treatment or control conditions so as to equate these groups for weight on Day 4.

Shock treatment. Each rat was separately placed in the treatment chamber for 4 min on each of 5 consecutive days. Half the rats of each sex received a shock treatment that occupied the last $3 \mathrm{~min}$ of this period and consisted of an uninterrupted presentation of scrambled shock. The other half of each sex served as nonshock-treated controls. For shock-treated subjects, an effort was made to obtain a gross indication of subject reactivity to shock during the course of treatment. On each treatment day, two experimenters employed an observation procedure that involved dividing each 3 -min shock into six $30-\mathrm{sec}$ segments. During each of these periods, both experimenters tallied the occurrence of events in either of two categories: one involving discernible vocalization and/or movement by the subject, and the other pertaining to ammeter fluctuations in excess of $0.3 \mathrm{~mA}$. Interrater agreement for these measures was $85 \%$ or better. After each 4-min period, the subject was removed to a holding cage and the chamber was thoroughly cleaned, dried, and checked for current leakage. A 1-week recovery period was interpolated between shock treatment and fighting tests.

Testing. The subjects in this study were paired within groups on a random basis, and the same pair was tested for shockelicited fighting at approximately the same time on each of 5 consecutive days. On each day, testing began $15 \mathrm{sec}$ following placement in the chamber and consisted of three consecutive test series. Each series was composed of 10 pseudo (i.e., no shock) and 10 actual shock presentations. The purpose of the pseudoshock presentations was to determine degree of fighting to the cues accompanying presentation of shock. No fighting was observed during these pseudopresentations, however. Each actual shock presentation was $0.5 \mathrm{sec}$ in duration, and the interval between shocks was $1.5 \mathrm{sec}$.

Scoring. Fighting was scored by a single experimenter who was uninformed about a subject's treatment history as well as whether a given presentation was a pseudo or an actual shock trial. The onset of each shock (either pseudo or actual) was indicated to the scorer by an unobtrusive visual signal. At that time, a fight response was scored if one or both rats attacked or made physical contact with one another by means of either the paws or the teeth. A posture response was scored if both subjects faced each other in an upright position but did not make obvious physical contact after light onset. To evaluate the reliability of these scoring procedures, three undergraduate students were instructed in their use, and all independently evaluated six pairs of rats. The scoring protocols were correlated for these students, and all interrater agreement values exceeded $90 \%$ for each of the six pairs observed.

\section{RESULTS}

\section{Treatment}

Examination of the tallies produced by the observation procedure employed on each of the 5 shock treatment days indicated that, regardless of sex, shock-treated subjects exhibited decreasing reactivity to shock in both categories during the course of each 3-min shock presentation. Furthermore, this apparent decrease in responding across the six $30-\mathrm{sec}$ portions of the treatment shock was observed on each of the 5 consecutive days. Invariably, on each day, shock treatment subjects reacted vigorously at the onset of shock but were virtually unresponsive during the final minute. In order to determine whether the apparent decrease in the frequency of tallies across segments of a treatment shock was reliable, Cochran's (1950) $Q$ tests were applied to the data from each category on each treatment day, disregarding sex. All $Q$ values on each day for each category exceeded 70.0, $\mathrm{p}<.001$. Moreover, the magnitude of the $Q$ values systematically increased for each category over days. This progressive increase in $Q$ values is consistent with the observation that the decrements in reactivity noted during the course of each shock became more rapid over treatment days.

\section{Test Data}

The effects of the independent variables in this study are most clearly revealed by the median number of fights per day for each group, depicted in Figure 1. These data reveal that non-shock-treated controls fought more than their shock-treated counterparts, regardless of sex. Also, it is apparent that males generally fought more than females, 


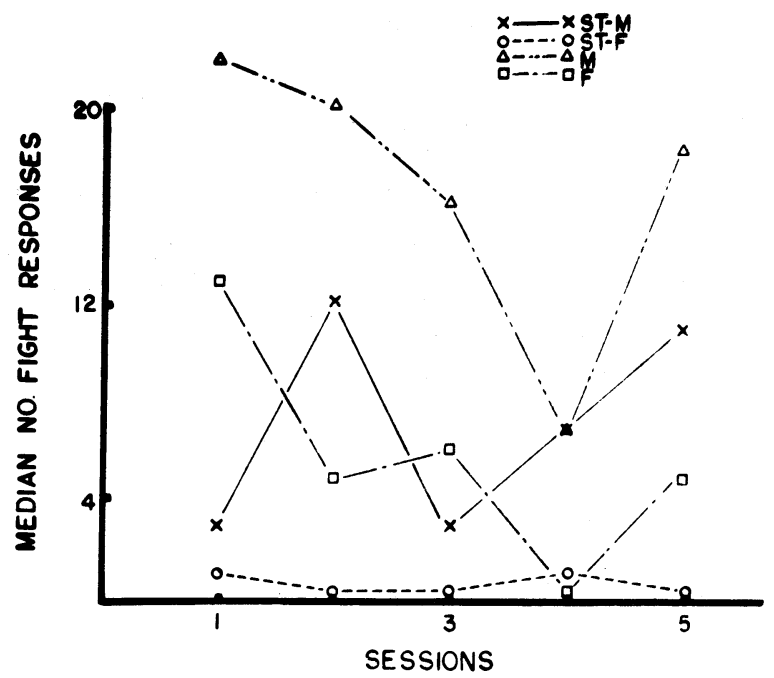

Figure 1. Median number of fight responses to test shocks, by groups, for each of five test sessions. Groups ST-M and ST-F were shock-treated males and females. Groups $M$ and $F$ were nonshock-treated males and females.

irrespective of whether shock treated or not. Furthermore, non-shock-treated controls exhibited a decreasing tendency to fight over the first 4 consecutive test days.

Both median and mean number of fights per day for each group were examined statistically. Since the conclusions emerging from these efforts were the same for either analysis, only the parametric comparisons of group means are reported here. An analysis of variance was performed involving both shock treatment and sex as between-subject factors and involving test days as a within-subject factor. The main effects of sex, $F(1,16)=4.6, p<.05$, days, $\mathrm{F}(4,64)=4.6, \mathrm{p}<.01$, and the Shock Treatment by Days interaction, $F(4,64)=7.2, p<.01$, were the only factors to emerge significant from this analysis. This latter interaction prompted evaluation of the main effect of shock treatment separately on each of the 5 consecutive days. Comparisons of shock-treated subjects with controls proved to be significant for Test Days 1-3, $\mathrm{p}<.05$, but not for Test Days 4 and 5 .

\section{DISCUSSION}

The results of this study indicate that both exposure to shock treatment as well as gender of the subject are important determinants of the degree of shock-elicited fighting obtained in albino rats. In general, male rats were found to fight more frequently in response to test shock than females and shocktreated subjects were observed, on the first few test days, to fight less frequently than nonshock controls, irrespective of sex.
At first glance, it would appear that the sex-related fighting differences obtained in this experiment contradict results reported by Milligan et al. (1973) and Powell et al. (1970) for albino rats. It should be noted, however, that although reliable gender differences in fighting behavior did not emerge from those studies, their results did reveal arithmetically more fighting in males than in females. Thus, at least the direction of the sex differences obtained both in the present study and in the earlier reports are the same. The fact that the sex-related differences in fighting obtained in the present study were significant supports the view that the earlier failures to find gender differences in the fighting of albino rats may be attributed, at least in part, to methodological details such as chamber size, shock intensity, and intershock interval during testing. This possible dependence of gender effects upon certain situational variables emphasizes the need to investigate systematically the effectiveness of various methodological details in determining sex-related differences in the degree of fighting behavior in rats.

Besides demonstrating reliable sex-related fighting differences, the present study represents the first systematic demonstration of the debilitating effects of prior shock treatment on the fighting behavior of both male and female rats. This finding importantly indicates that the generality of shock-treatment effects may be extended not only to different strains (Anderson \& Packham, 1967) and different species of organisms (Braud, Wepmann, \& Russo, 1969; Overmier \& Seligman, 1967), but also to different genders within the same species. It is also important to note that the attenuation of fighting behavior in both males and females produced by prior exposures to inescapable shock occurred despite a rather substantial initial difference in the fighting of these genders as revealed by comparison male and female controls on Test Day 1.

Results of the present study also contain clues regarding the possible mechanism underlying the debilitating effects of exposure to inescapable shock. The measures of responsivity to treatment shock in the present study uniformly changed during the course of each treatment shock in the direction of decreased reactivity. Furthermore, it was noted that the tendency to refrain from movements during shock occurred earlier and earlier over the 5 treatment days. These observations are consistent with (although not definite support for) the view that the rats in this study acquired a tendency to become immobile in the presence of treatment shock. The reinforcement for this alleged learning is not clear, but may have involved pain reduction achieved through postural adjustment. In any case, if conditions were present during inescapable shock treat- 
ment that promoted the learning of immobility reactions in the presence of shock cues, one might expect such postural adjustments to generalize to the shock cues present in the testing situation and therein to compete with the active, fighting behavior normally evoked by such shock. Control subjects, on the other hand, would not have had the opportunity to acquire such competing behaviors during the treatment phase and therefore would be expected to exhibit relatively normal levels of fighting in response to test shock. Thus, the gross correlation between reduced responsivity to treatment shock and attenuated fighting in response to test shock in the present study is consistent with a general view known as the competing response interpretation of shock-treatment effects (see Anderson, Cole, \& McVaugh, 1968, Payne et al., 1970, and Powell et al., 1972, for a more detailed exposition of this view).

It is necessary to emphasize that, although the above noted correlation between behavior during treatment shock and behavior during test shock is suggestive of a competing response mechanism, the present evidence cannot be regarded as definitive in this regard. The failure to manipulate systematically the type of behavior that may have been learned during treatment shock in the present study leaves open the possibility that both the attenuated movement during treatment shock as well as the reduced fighting behavior during test shock are products of some other process(es). One alternative process that could be invoked to account for the present findings is adaptation or habituation to shock. According to this view, diminished receptor and/or effector activity in ST groups resulting from prolonged exposure to shock during treatment could reduce reactivity and fighting in response to shock. Another mechanism that may explain the present findings is that of learned helplessness. According to this view, exposure to inescapable shock leads to reduced fighting, not because specific responses are acquired that prove to be incompatible with behaviors in the test situation, but rather because under conditions of inescapable shock organisms are believed to acquire a "cognitive set" to the effect that "nothing they do matters." That is, organisms exposed to inescapable shock alledgedly learn that their behavior in no way controls termination of shock, and as a result are believed to suffer subsequent associative and motivational deficits (see Maier \& Seligman, 1976). Further research is required to determine the accuracy of these respective views concerning the effects of inescapable shock on the magnitude of subsequent shock-elicited fighting behavior in rats.

\section{REFERENCES}

Anderson, D. C., Cole, J., \& $\mathrm{MCV}_{\text {Augh, W. Variations in }}$ unsignalled inescapable preshock as determinants of responses to punishment. Journal of Comparative and Physiological Psychology, 1968, 65, Monograph Supplement, No. 3, Part 2.

Anderson, D. C., Murcurio, J., \& Mahoney, P. Inhibition of preshock-produced reflexive fighting by a $39 \mathrm{KHz}$ tone. Physiology and Behavior, 1970, 5, 577-581.

Anderson, D. C., \& Packham, S. Preshock, scopolamine, and genetic strain as determinants of the effects of punishment. Psychonomic Science, 1967, 9, 49-50.

Braud, W., Wepmann, B., \& Russo, D. Task and species generality of the "helplessness" phenomenon. Psychonomic Science, 1969, 16, 154-155.

Campbell, B. A., \& Teghtsoonian, R. Electrical and behavioral effects of different types of shock stimuli on the rat. Journal of Comparative and Physiological Psychology, 1958, 51, 185-192.

COCHRAN, W. G. The comparison of percentages in matched samples. Biometrics, 1950, 37, 256-266.

Connor, R. L., \& Levine, S. Hormonal influences on aggressive behavior. In S. Garattini \& E. B. Sigg (Eds.), Aggressive behavior. New York: Wiley, 1969.

Hutchinson, R. R., Ulrich, R. E., \& Azrin, N. H. Effects of age and related factors on the pain-aggression reaction. Journal of Comparative and Physiological Psychology, 1965, 59, 365-369.

Maier, S. F., Anderson, C., \& Lieberman, D. A. Influence on control of shock on subsequent shock-elicited aggression. Journal of Comparative and Physiological Psychology, 1972, 81, 94-100.

Maier, S. F., \& Seligman, M. E. P. Learned helplessness: Theory and evidence. Journal of Experimental Psychology: General, 1976, 105, 3-46.

Milligan, W. L., Powell, D. A., \& Borasio, G. Sexual variables and shock-elicited aggression. Journal of Comparative and Physiological Psychology, 1973, 83, 441-450.

Overmier, J. B., \& Seligman, M. E. P. Effects of inescapable shock upon subsequent escape and avoidance responding. Journal of Comparative and Physiological Psychology, 1967, 63, 28-33.

Payne, R., Anderson, D. C., \& Murcurio, J. Preshockproduced alterations in pain elicited fighting. Journal of Comparative and Physiological Psychology, 1970, 71, 258-266.

Powell, D. A., \& CREER, T. L. Interaction of developmental and environmental variables in shock-elicited aggression. Journal of Comparative and Physiological Psychology, 1969, 69, 219-225.

Powell, D. A., Francis, M. J., Francis, J., \& Schneiderman, N. Shock-induced aggression as a function of prior experience with avoidance, fighting, or unavoidable shock. Journal of the Experimental Analysis of Behavior, 1972, 18, 323-332.

Powell, D. A., Silverman, T., Francis, J., \& Schneiderman, $\mathrm{N}$. The effects of sex and prior experience with fighting on shockelicited aggression. Communications in Behavioral Biology, 1970, 5, 51-56.

TondAT, L. M. Is the effect of preshock treatment on shock-elicited aggression independent of situational stimuli? Psychological Record, 1974, 24, 409-417.

UlRich, R., \& Azrin, N. H. Reflexive fighting in response to aversive stimulation. Journal of the Experimental Analysis of Behavior, 1962, 5, 511-520.

Ulrich, R. E., Hutchinson, R. R., \& Azrin, N. H. Pain-elicited aggression. The Psychological Record, 1965, 15, 111-126.

(Received for publication April 7, 1977; revision accepted September 1, 1977.) 